\title{
甲烷干气重整镍基复合结构催化剂的研究进展
}

\author{
张鹏, 张晴, 刘 静, 高 濂 \\ (上海交通大学 材料科学与工程学院, 上海 200240)
}

摘 要: 甲烷干气重整反应能够实现温室气体 $\mathrm{CO}_{2}$ 和 $\mathrm{CH}_{4}$ 的转化利用, 其反应产物合成气可以通过费托反应进一步 生产液态燃料，该反应在能源与环境领域具有重要意义。寻找合适的催化剂是推动甲烷干气重整工业化的关键。镍 基复合结构催化剂因其与贵金属催化剂相媲美的催化活性和低廉的工业成本而受到广泛关注，但镍基催化剂存在 高温下长时间反应后碳沉积和金属组分烧结所导致的失活问题，严重影响了其工业应用和干气重整化工的发展。本 文从镍基复合结构催化剂的成分、结构、制备方法及模拟计算设计等方面出发，介绍了改进镍基催化剂活性、抗积 碳和抗烧结性能的研究进展, 并结合最新的原子催化以及原位表征等研究进展对干气重整研究的发展趋势进行 展望。

关 键 词: 甲烷干气重整; 镍基催化剂; 抗积碳; 金属组分烧结; 研究进展

中图分类号: O643 文献标识码: A

\section{Research Progress of Ni-based Composite Catalysts for Methane Dry Reforming}

\author{
ZHANG Peng, ZHANG Qing, LIU Jing, GAO Lian
}

(School of Material Science and Engineer, Shanghai Jiao Tong University, Shanghai 200240, China)

\begin{abstract}
Methane Dry Reforming (DRM) catalysis converts the two greenhouse gases, $\mathrm{CO}_{2}$ and $\mathrm{CH}_{4}$ into syngas, which can be further utilized for liquid fuels production through Fischer-Tropsch synthesis. The DRM reaction is therefore of significance for both energy and environments. The key issue to promote industrialization of DRM reaction is to develop suitable catalysts. Nickel based composite catalysts have received extensive attention due to their outstanding catalytic activities comparable to those of precious metals and their bargain price. However, nickel based catalysts usually undergo severe deactivation due to the coke deposition and metal sintering during long-term reaction at high temperature, which seriously limit their industrial applications and development of the engineering of DRM. Many researches have been carried out to address this issue. In this review, the recent research progress in the activity, coke resistance, and sintering resistance of Ni-based composite catalysts are introduced from the aspect of catalyst components, structures, preparation methods, and simulations. The research trends of DRM catalysts are also predicted based on the recent research progress in single-atomic catalysis and the in-situ characterization techniques for catalysis.
\end{abstract}

Key words: methane dry reforming; Ni-based catalyst; coke resistance; metal sintering; research progress

现代工业的急速发展和石油燃料的快速消耗使 得大气中温室气体二氧化碳 $\left(\mathrm{CO}_{2}\right)$ 的浓度迅速上升,
引发的温室效应带来了全球性气候变暖、海平面上 升和土地沙漠化等环境和气候问题 ${ }^{[1-3]}$ 。据《自然》

收稿日期：2017-12-07; 收到修改稿日期：2018-03-04

基金项目: 国家自然科学基金(51672174，51502170); 上海市自然科学基金(17ZR1414900); 上海市科委材料基因组研究计 划(16DZ2260602)

National Natural Science Foundation of China (51672174, 51502170); Natural Science Foundation of Shanghai Municipality (17ZR1414900); The Material Genome Initiative Project Foundation of Science and Technology Commission of Shanghai Municipality (16DZ2260602)

作者简介: 张 鹏(1976-), 男, 教授. E-mail: pengzhang2010@sjtu.edu.cn 
杂志近期报道, “全球碳计划”组织(Global Carbon Project)在联合国气候变化会议上的研究报告指出, 2017 年化石燃料与工业排放的二氧化碳打破过去 三年维持零增长的局面, 预计将达到 370 亿吨的新 纪录, 与 2016 年相比提高了 $200 \%$, 排放增长的主 要原因是中国境内工业生产的增加以及水力发电的 减少 ${ }^{[4]}$ 。亟需有效的方法实现二氧化碳的消除和转化。

甲烷 $\left(\mathrm{CH}_{4}\right)$ 是一种比二氧化碳更严重的温室气 体 $^{[3,5]}$, 但又是可以替代石油和煤炭的储量丰富、价 格低廉并且相对清洁的燃料及化学工业原料。自然 界中, 甲烷分布广泛, 主要以天然气、可燃冰、石油 气和煤层气等不同形式存在。美国是目前天然气化 工最发达的国家, 其天然气生产和化工也大大减少 了石油的用量。近年来由于全球页岩气的大规模勘 探开采, 甲烷的工业利用已成为学术界和产业界研 究的重点 ${ }^{[6-7]}$ 。甲烷干气重整反应(Dry Reforming of Methane, DRM, $\mathrm{CH}_{4}+\mathrm{CO}_{2} \rightarrow 2 \mathrm{H}_{2}+2 \mathrm{CO}, \Delta H_{298}=+$ $247 \mathrm{~kJ} / \mathrm{mol}$ ) 能够将甲烷和二氧化碳转化为合成气 (氢气和一氧化碳混合气)。由于产物中 $\mathrm{H}_{2} / \mathrm{CO}$ 比值 低, 与传统的水蒸气重整(Steam Reforming of Methane, $\mathrm{SRM}, \mathrm{CH}_{4}+\mathrm{H}_{2} \mathrm{O} \rightarrow 3 \mathrm{H}_{2}+\mathrm{CO}, \Delta H_{298}=+206 \mathrm{~kJ} / \mathrm{mol}$ )相 比更适合通过费托反应(Fischer-Tropsch Synthesis) 或羰基合成生产烃类、甲醇和其他液体燃料 ${ }^{[8-11]}$ 。 由于这一反应能够有效转化利用两种温室气体, 同 时生产化工原料, 因此在能源与环境领域都受到了 广泛关注 ${ }^{[11-12]}$ 。中国拥有极丰富的天然气、页岩气 及可燃冰资源储量, 所以甲烷重整技术对中国的能 源战略也具有重要意义。

\section{1 甲烷干气重整及催化剂研究概述}

\section{1 甲烷干气重整反应}

甲烷干气重整过程涉及复杂的反应体系，重整 过程中主要的化学反应及其热力学数据如表 1 所 示。其中反水气反应(Reverse Water Gas Shift Reaction, RWGS) 是甲烷干气重整过程中的主要副反应, 会导 致产物中 $\mathrm{H}_{2} / \mathrm{CO}$ 比值降低。一氧化碳歧化(Boudouard Reaction)和甲烷裂解(Methane Cracking)两个副反应
会造成积碳, 从而影响催化剂性能。Wang 等 ${ }^{[13]}$ 给出 了这些反应的吉布斯自由能公式(表 1), 并以 $\triangle G=0$ 作为反应临界条件, 分别得出了各反应的极限温 度：主反应甲烷二氧化碳重整反应的最低反应温度 为 $913 \mathrm{~K}\left(640^{\circ} \mathrm{C}\right)$, 反水气反应的最高反应温度为 $1093 \mathrm{~K}\left(820^{\circ} \mathrm{C}\right)$, 一氧化碳歧化反应的最高反应温度 为 $973 \mathrm{~K}\left(700^{\circ} \mathrm{C}\right)$, 甲烷裂解反应的最低反应温度为 $830 \mathrm{~K}\left(557^{\circ} \mathrm{C}\right)$ 。

Zhang 等 ${ }^{[14]}$ 则通过热力学计算得到以上反应的 热力学平衡常数随温度变化的情况, 如图 1 所示。 其中主反应甲烷干气重整为剧烈吸热反应, 反应平 衡常数随温度升高显著增大, 因此高温条件有利于 反应的进行; 甲烷裂解和反水气反应为中度吸热反 应，反应平衡常数同样随温度升高而增大; 一氧化碳 歧化反应和反碳气化反应(Reverse Carbon Gasification Reaction, $2 \mathrm{CO} \rightarrow \mathrm{CO}_{2}+\mathrm{C}, \Delta H_{298}=-131 \mathrm{~kJ} \cdot \mathrm{mol}^{-1}$ )为放 热反应, 高温在热力学上不利于反应的进行。高温 (1023 K 及以上)更有利于主反应平衡转化率的增加, 因此在竞争反应体系下高温可以在一定程度上抑制 副反应发生。

目前甲烷干气重整反应研究的侧重点主要有两 方面: (1)设计具有高活性、抗积碳和抗烧结性能的 新型催化剂; (2)通过动力学方法, 对甲烷干气重整 机理进行细致深入的研究, 从而对重整反应有进一 步的认识。

\section{2 甲烷干气重整催化剂}

甲烷干气重整催化剂一般是由载体和催化活性 金属组成的复合结构, 其中催化剂的活性金属是催 化剂最重要的组分。一般来说, $\mathrm{CH}_{4}$ 只能在金属表面 发生吸附并解离生成 $\mathrm{H}$ 原子和 $\mathrm{CH}_{x}(x=0 \sim 4), x$ 的值 与金属基底和反应温度有关 ${ }^{[12]} 。 \mathrm{CH}_{x}$ 和 $\mathrm{H}$ 原子能够 吸附在金属活性位点上, $\mathrm{H}$ 物种会重新结合生成 $\mathrm{H}_{2}$ 分子, 并以气态形式释放出来 ${ }^{[15]}$ 。

甲烷干气重整催化剂可以根据活性金属组分分 为贵金属催化剂和非贵金属催化剂两类。其中贵金 属催化剂以 $\mathrm{Rh} 、 \mathrm{Ru} 、 \mathrm{Pt} 、 \mathrm{Pd}$ 和 Ir 等作为催化剂活 性金属组分，表现出较高的反应活性，长时间反应 后仍能保持良好的稳定性和优异的抗积碳性能 ${ }^{[16-17]}$, 因此许多工作都是围绕贵金属催化剂的研究展开。但

表 1 甲烷干气重整过程中主要化学反应 ${ }^{[12-13]}$

Table 1 Main chemical reactions during methane dry reforming process ${ }^{[12-13]}$

\begin{tabular}{ccccc}
\hline Reaction & Reaction equation & $\Delta G$ & $\Delta H_{298} /\left(\mathrm{kJ} \cdot \mathrm{mol}^{-1}\right)$ & Limit temperature $/ \mathrm{K}$ \\
\hline DRM & $\mathrm{CH}_{4}+\mathrm{CO}_{2} \rightarrow 2 \mathrm{H}_{2}+2 \mathrm{CO}$ & $61770-67.32 T$ & 247.3 & 913 \\
RWGS & $\mathrm{CO}_{2}+\mathrm{H}_{2} \rightarrow \mathrm{CO}+\mathrm{H}_{2} \mathrm{O}$ & $-8545+7.84 T$ & 41.0 & 1093 \\
Boudouard reaction & $2 \mathrm{CO} \rightarrow \mathrm{CO}_{2}+\mathrm{C}$ & $-39810+40.87 T$ & -171.0 & 973 \\
Methane cracking & $\mathrm{CH}_{4} \rightarrow \mathrm{C}+2 \mathrm{H}_{2}$ & $2190-26.45 T$ & 75.0 & 830 \\
\hline
\end{tabular}




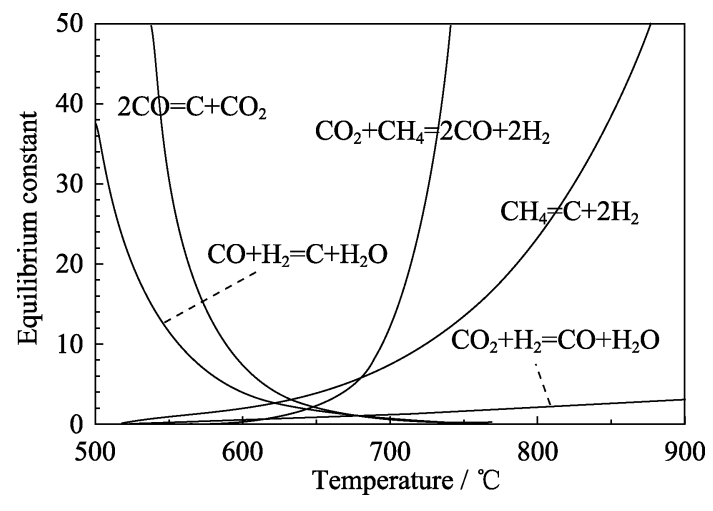

图 1 甲烷干气重整过程中各反应平衡常数随温度变化曲线 ${ }^{[14]}$

Fig. 1 Equilibrium constants of reactions as a function of temperature during $\mathrm{DRM}^{[14]}$

贵金属存在储量有限且价格昂贵的问题，实际工业 生产的成本较高，因此研究人员希望寻找性能优良 的非贵金属催化剂作为替代。用于甲烷干气重整的非贵 金属主要包括第 IB 族的 $\mathrm{Cu}$ 和 VIII 族的 $\mathrm{Fe} 、 \mathrm{Co}$ 和 $\mathrm{Ni}$ 。

\section{3 镍基催化剂及其失活}

在诸多非贵金属催化剂中, 镍基催化剂表现出 与贵金属催化剂相謧美的优秀催化活性，且金属镍 储量丰富, 价格低廉, 受到了广泛关注 ${ }^{[18-22]}$ 。然而和 贵金属催化剂相比，以镍基催化剂为代表的非贵金 属催化剂往往更容易在长时间高温反应下失活。造 成镍基催化剂失活的主要因素有以下两点:

(1)积碳

催化剂表面积碳是造成镍基催化剂失活的最主 要原因, 甲烷干气重整反应的原料 $\mathrm{CH}_{4}$ 和 $\mathrm{CO}_{2}$ 均为 含碳气体，长时间高温反应会由于甲烷裂解和一氧 化碳歧化等副反应而形成沉积在金属粒子表面的碳 单质 ${ }^{[16]}$ 。积碳的种类可根据形貌划分为无定形碳、 聚合碳、碳纳米管、石墨化碳及丝状碳等 ${ }^{[23]}$ 。其中 无定形碳又被称为 $\mathrm{C}_{\alpha}$, 由吸附在金属活性中心的碳 原子组成，具有最高的反应活性，在 $100^{\circ} \mathrm{C}$ 就可以 被氧化消除; 聚合碳由部分氢化的碳-碳链组成, 因 此与无定形碳相比反应活性相对较低, 其氧化温度 随着 $\mathrm{H}: \mathrm{C}$ 比值的降低而上升。无定形碳与聚合碳等 属于能在温和条件下氧化消除的碳物种，不会阻挡 催化剂表面活性位点 ${ }^{[24]}$ 。石墨化碳由六个碳原子形 成的环状结构组成, 具备最低的反应活性, 需要很 高的反应温度才能氧化消除。碳纳米管和丝状碳同 样属于低反应活性的积碳物种。这类难以消除的碳 物种会阻碍催化剂表面活性位点和反应气体接触, 堵塞催化剂空隙, 导致催化剂活性逐渐下降甚至完 全失活 ${ }^{[9,20,24]}$ 。

催化剂表面沉积的碳物种一般能通过碳消除反 应 $\mathrm{CO}_{2}+\mathrm{C} \rightarrow 2 \mathrm{CO}$ 被 $\mathrm{CO}_{2}$ 氧化消除, 因此催化剂积
碳程度是由甲烷裂解和/或一氧化碳歧化与碳消除 反应之间的平衡所决定 ${ }^{[25]}$, 可以从抑制碳沉积反应 和促进碳消除反应两个角度出发, 通过合理的催化 剂设计提高催化剂抗积碳性能。研究表明，活性金 属在催化剂载体表面的高分散性能够降低其团聚尺 寸, 进而有效限制碳沉积 ${ }^{[16,26-27]}$; 同样有研究认为, 只有金属活性组分的尺寸大于一定临界尺寸时 $(\geqslant 9 \mathrm{~nm})$ 碳单质才能形核 ${ }^{[9]}$, 因此通过改变催化剂载体、活性 金属组分、催化剂助剂和制备方法等诸多因素对催 化剂活性金属的分散性和颗粒尺寸进行调控，可以 有效抑制碳沉积反应发生，进而提高抗积碳性能 ${ }^{[28]}$ 。 此外, 添加合适的助剂增强催化剂表面碱性能提高 表面 $\mathrm{CO}_{2}$ 吸附, 促进沉积碳物种的氧化消除, 从促 进碳消除反应进行的角度来增强催化剂的抗积碳性。

(2)活性组分烧结

催化剂中金属活性组分在高温下烧结聚集也是 造成催化剂活性逐渐下降的原因。负载在载体上的 小尺寸金属晶粒具有较大的表面能，因而热力学上 小晶粒存在自发团聚并有降低表面能的趋势 ${ }^{[29]}$ 。当 催化剂在高温下长时间反应时，金属颗粒会在载体 表面迁移并逐渐由小晶粒聚集成大晶粒，导致催化 活性组分的比表面积降低、反应活性位点数量下降, 从而导致催化活性下降甚至失活, 这一现象即为催 化剂的烧结。影响催化剂活性组分烧结的主要因素 是金属颗粒与载体间的相互作用力，因此增强金属 与载体间的接触强度是抑制烧结的有效方法。另外, 金属组分在催化剂表面的迁移还受到催化剂载体结 构的制约，通过改良载体结构同样可以限制烧结。

除了上述两个因素外，镍基催化剂在高温下长 时间反应，还容易和载体发生不可逆转的反应(如 生成活性差的尖晶石结构), 这些问题严重阻碍了 镍基催化剂在实际工业生产中的应用。研发出具有 优良抗积碳性能和高温稳定性的镍基催化剂, 推动 甲烷干气重整反应的工业化运行，已经成为这一领 域的研究热点。

\section{2 镍基复合结构催化剂活性及稳定性 的提高}

针对镍基催化剂普遍存在的积碳和活性组分烧 结的问题, 研究人员利用各种金属活性组分、载体 结构、助剂添加、合成方法和 DFT 模拟计算等，设 计制备出具有优异催化活性和稳定性的镍基催化剂。

\section{1 金属一载体相互作用力}

增强金属-载体相互作用力可以同时提高催化 
剂的活性和稳定性。在同样的镍负载量下, 镍组分 和载体之间的强相互作用力可以得到稳定的小尺寸 $\mathrm{Ni}$ 单质，提高 $\mathrm{Ni}$ 金属的活性表面积，从而提供更多 反应位点, 提高催化剂转化率。此外, 研究认为只有 金属活性组分的尺寸大于临界尺寸 $9 \mathrm{~nm}$ 时才会发 生积碳 ${ }^{[9]}$, 足够小的活性组分尺寸和高分散度可以 有效抑制碳纤维的形核和生长。因此增强金属-载体 相互作用力，限制 $\mathrm{Ni}$ 金属颗粒在高温还原和催化过 程中的颗粒生长, 能同时提高催化剂抗烧结性和抗 积碳性能。

近些年来, 许多研究者使用具备有序矿物晶体 结构的含 $\mathrm{Ni}$ 氧化物作为前驱体 (如钙钛矿结构 $\mathrm{ABO}_{3}{ }^{[30-31]}$, 尖晶石结构 $\mathrm{ABO}_{4}$ 和 $\mathrm{A}_{2} \mathrm{BO}_{4}{ }^{[21,32]}$, 六铝 酸盐 $\mathrm{AB}_{y} \mathrm{Al}_{12-y} \mathrm{O}_{19-\delta}{ }^{[33]}$ 和固溶体等 ${ }^{[34-35]} ; \mathrm{B}=\mathrm{Ni}$ ) 来得 到具有强金属-载体相互作用力的小尺寸 $\mathrm{Ni}$ 纳米颗 粒。Ni 元素在这些有序晶体中呈均匀分布, 由于晶 格结构对纳米颗粒的生长和迁移的限制作用, 在还 原气氛中经过固相脱溶反应后能够得到尺寸较小、 分布均匀且和载体结合紧密的 $\mathrm{Ni}$ 金属单质, 从而提高 了镍基催化剂的抗烧结和抗积碳性能 ${ }^{[20,36-37]}$ 。本课题 组 ${ }^{[21]}$ 通过 NiAl-LDHs 制备了具有多级空心球结构 的催化剂 $\mathrm{Ni} / \gamma-\mathrm{Al}_{2} \mathrm{O}_{3}$, 研究了前驱体煅烧温度对于 金属-载体相互作用力的影响。氧化态前驱体的 $\mathrm{TPR}$ (图 2)结果显示, $400^{\circ} \mathrm{C}$ 煅烧的样品仅有一个位 于 $450 \sim 500^{\circ} \mathrm{C}$ 附近的还原峰, 归属于与氧化物载体 有弱接触作用的 $\mathrm{NiO}$ 物种; 600 和 $800^{\circ} \mathrm{C}$ 㷽烧后样品 的 TPR 曲线中还存在位于 $800^{\circ} \mathrm{C}$ 附近的高温还原峰, 是来自于与载体有强相互接触作用的尖晶石相 $\mathrm{NiAl}_{2} \mathrm{O}_{4}$, 但在 $600^{\circ} \mathrm{C}$ 煅烧的样品中高温还原峰强度 非常微弱。 $800^{\circ} \mathrm{C}$ 㷽烧的样品在还原后得到了分布均 匀的小尺寸 $\mathrm{Ni}$ 纳米颗粒; 低温烣烧的两个样品中观

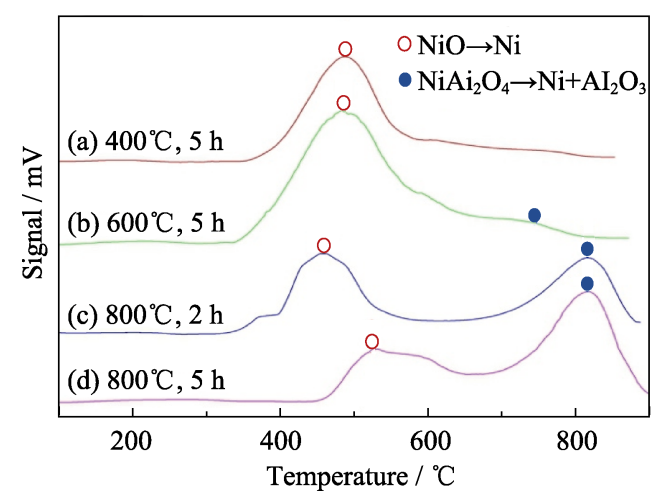

图 2 不同㷽烧温度和㷽烧时间的 $\mathrm{NiO}-\gamma-\mathrm{Al}_{2} \mathrm{O}_{3}$ 样品的程序 升温还原(TPR)曲线 $[21]$

Fig. 2 Temperature programmed reduction (TPR) curves of $\mathrm{NiO}-\gamma-\mathrm{Al}_{2} \mathrm{O}_{3}$ calcined at different temperatures for different durations $^{[21]}$
察到了粒径 $>50 \mathrm{~nm}$ 的颗粒和许多小的孔洞, 表明 还原过程中存在金属迁移和烧结现象。由尖晶石相 为前驱体的催化剂表现出了最高的催化活性, 且经 过 $50 \mathrm{~h}$ 催化后没有烧结或者积碳现象产生, 显示出 了优越的稳定性。此外, 还通过一步水热法制备了 硅酸镍有序晶格结构的前驱体, 还原后得到具备强 金属-载体相互作用力和高比表面积的 $\mathrm{Ni} / \mathrm{SiO}_{2}$ 空心 球多级结构催化剂 ${ }^{[38]}$ 。

最近, $\mathrm{Li}$ 等 ${ }^{[31]}$ 对比了 $\mathrm{La}_{2} \mathrm{O}_{2} \mathrm{CO}_{3}$ 纳米棒和 $\mathrm{La}_{2} \mathrm{O}_{3}$ 载体通过浸渍法制备的 $\mathrm{Ni}$ 基催化剂性能, 结果表明 $\mathrm{La}_{2} \mathrm{O}_{2} \mathrm{CO}_{3}$ 载体上的 $\mathrm{Ni}$ 颗粒更小, 金属活性表面积 更高, 不仅在 $500 \sim 700^{\circ} \mathrm{C}$ 温度范围展现出最佳的催 化活性, 且在 $700^{\circ} \mathrm{C}$ 下高空速催化 $50 \mathrm{~h}$ 后甲烷和二 氧化碳转化率仍保持稳定。催化反应 $50 \mathrm{~h}$ 后的样品 表征结果显示, 使用 $\mathrm{La}_{2} \mathrm{O}_{2} \mathrm{CO}_{3}$ 为载体前驱体增强 了 $\mathrm{Ni}$ 和 $\mathrm{La}_{2} \mathrm{O}_{3}$ 的相互作用力, 有效抑制了烧结; 高 分散的 $\mathrm{Ni}$ 颗粒降低了积碳的程度，同时抑制了能够 削弱催化剂性能的非纤维状碳的形成。

\section{2 双金属协同效应}

在镍基催化剂中加入贵金属或另一种过渡金属 是一种调控催化剂性能的有效方法 ${ }^{[39]}$ 。由于两种金 属间存在 “协同效应”，加入第二种金属组分会造成 催化剂几何效应和电子效应的改变, 影响催化反应 动力学, 可以得到比单金属催化剂更优越的催化活 性、选择性和稳定性。例如 $\mathrm{Wu}$ 等 ${ }^{[40]}$ 通过 DFT 模拟 证明在 $\mathrm{Ni}$ 基催化剂中加入 $\mathrm{Co}$ 能够改变 $\mathrm{CH}_{4}$ 的活化机 理: $\mathrm{CH}_{4}$ 在 $\mathrm{Ni}$ 表面活化是通过 $\mathrm{CH}_{4}+{ }^{*}+{ }^{*} \rightarrow \mathrm{CH}_{3}{ }^{*}+\mathrm{H}^{*}$ 实现的, 而在 $\mathrm{Co}$ 和 $\mathrm{Ni}-\mathrm{Co}$ 表面则通过 $\mathrm{CH}_{4}+{ }^{*}+\mathrm{O}^{*} \rightarrow$ $\mathrm{CH}_{3}{ }^{*}+\mathrm{HO}^{*}$ 完成, 这一现象被归因于 $\mathrm{Co}$ 的亲氧性。

在镍基催化剂中添加少量 $\mathrm{Ru} 、 \mathrm{Rh} 、 \mathrm{Pt}$ 和 $\mathrm{Pd}$ 等 贵金属能够有效地提高镍基催化剂的性能 ${ }^{[41-42]}$ 。 Chen 等 ${ }^{[41]}$ 在 $\mathrm{Ni}_{0.03} \mathrm{Mg}_{0.97} \mathrm{O}$ 固溶体内分别加入少量 的 Pt、Pd 和 Rh, 并对单金属催化剂和双金属催化 剂的性能进行了分析对比。 $\mathrm{Ni}_{0.03} \mathrm{Mg}_{0.97} \mathrm{O}$ 固溶体在 较低温度下 $(773 \mathrm{~K})$ 反应会由于 $\mathrm{Ni}$ 金属被氧化而失 活, 加入贵金属产生的协同效应能够有效提高 $\mathrm{Ni}$ 的 还原性，进而提高该催化剂的活性和稳定性。除传 统的金、银和铂系金属外, 稀有金属 Re 同样被用于 甲烷干气重整催化, 近期 Zubenko 等 ${ }^{[37]}$ 报道了一种 Re-Ni 合金负载型催化剂 LNRF，他们通过溶胶凝胶法将 $\mathrm{Ni}$ 和 $\mathrm{Re}$ 金属盐掺杂到 $\mathrm{LaFeO}_{3}$ 钙铁矿结构 中, Ni-Re 合金掺杂的催化剂表现出优秀的催化活 性和稳定性, 且在连续催化 $70 \mathrm{~h}$ 后仍没有发生碳沉 积。这是由于 $\mathrm{Re}$ 的掺杂增强了 $\mathrm{Ni}$ 纳米颗粒的活性, 并且通过固相原位脱溶生长得到的催化剂具备强金 属-载体相互作用力, 有效限制了碳沉积、纳米颗粒 
烧结以及含有 $\operatorname{Re}$ 的物相在高温下蒸发气化所带来 的催化剂失活。

尽管在镍基双金属催化剂中往往只添加少量贵 金属，但仍然会提升催化剂的生产成本，因此一些 研究者在镍基催化剂内掺杂了 $\mathrm{Cu} 、 \mathrm{Co} 、 \mathrm{Fe}$ 等其他 非贵金属以期降低工业化成本。本课题组 ${ }^{[22]}$ 通过水 热法制备了具有不同 $\mathrm{Cu} / \mathrm{Ni}$ 比值的 $\mathrm{Cu}-\mathrm{Ni} / \mathrm{SiO}_{2}$ 多级 结构催化剂, 研究了铜元素的加入量对不同反应温 度下甲烷转换率的影响(图 3)。与纯镍基催化剂 $\mathrm{Ni} / \mathrm{SiO}_{2}$ 相比, 少量 $\mathrm{Cu}$ 掺杂 $\left(\mathrm{CuNi}_{4} / \mathrm{SiO}_{2}\right)$ 对催化性能 无太大影响, 过量 $\mathrm{Cu}$ 掺杂 $\left(\mathrm{CuNi} / \mathrm{SiO}_{2}\right)$ 反而会导致 催化活性下降, 而适量的 $\mathrm{Cu}$ 元素 $\left(\mathrm{CuNi}_{3} / \mathrm{SiO}_{2}\right)$ 不仅 能提高催化活性, 还能提高氢气选择性。过量 $\mathrm{Cu}$ 元 素带来的负面影响可能来自于 $\mathrm{Cu}$ 原子的表面富集, 其表面自由能 $\sigma_{(111) \mathrm{Cu}}=69.5 \mathrm{~kJ} / \mathrm{mol}$ 低于镍原子表面 自由能 $\sigma_{(111) \mathrm{Ni}}=80 \mathrm{~kJ} / \mathrm{mol}$, 因此 $\mathrm{Cu}$ 原子在还原过程 中更倾向于向表面移动, 使得 $\mathrm{Ni}$ 原子在表面所占比 例降低，导致催化活性下降。Zhang 等 ${ }^{[14]}$ 用共沉淀 法制备了一系列 $\mathrm{Ni}-\mathrm{Me}(\mathrm{Me}=\mathrm{Co} 、 \mathrm{Fe} 、 \mathrm{Cu}$ 或 $\mathrm{Mn})$ 双 金属催化剂, 并对比研究了催化甲烷干气重整的性 能。和其他 Ni-Me 组合相比, Ni-Co 双金属催化剂表 现出最优越的催化活性和稳定性, 可以将其归因于双 金属效应、良好的金属分散性、较大的金属表面积、 强金属-载体相互作用力及不同类型固溶体的形成。

\section{3 催化剂助剂}

对于甲烷干气重整反应催化剂, 催化剂表面的 酸碱性对于碳沉积有重要影响。Horiuchi 等 ${ }^{[43]}$ 认为, 碱性金属氧化物能够增强对 $\mathrm{CO}_{2}$ 的吸附能力, 生成 更多的活性氧原子 $\left(\mathrm{O}_{\mathrm{ad}}\right) ; \mathrm{O}_{\mathrm{ad}}$ 能够通过 $\mathrm{CH}_{\mathrm{x}, \mathrm{ad}}+\mathrm{O}_{\mathrm{ad}} \rightarrow$ $\mathrm{CO}+\mathrm{H}_{2}$ 反应有效防止 $\mathrm{CH}_{\mathrm{x}, \mathrm{ad}}$ 在 $\mathrm{Ni}$ 金属活性中心上

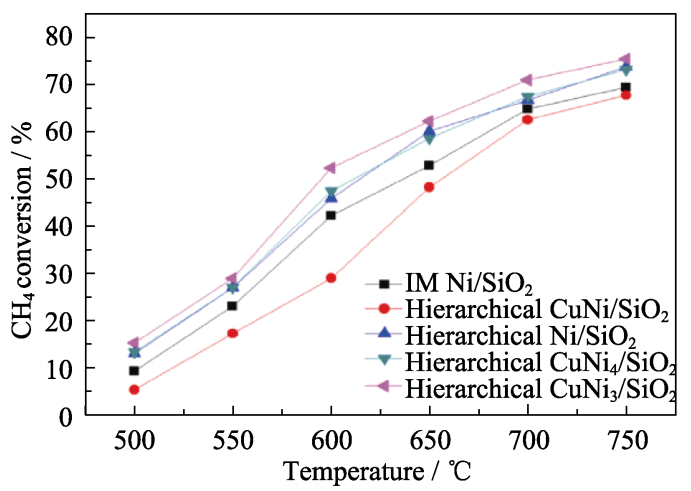

图 3 具有不同 $\mathrm{Cu} / \mathrm{Ni}$ 比值的 $\mathrm{Cu}-\mathrm{Ni} / \mathrm{SiO}_{2}$ 催化剂及对照样 $\mathrm{IM}$ $\mathrm{Ni} / \mathrm{SiO}_{2}$ 在不同温度下用于甲烷干气重整反应的甲烷转化率 ${ }^{[22]}$

Fig. 3 Methane conversion of the methane dry reforming catalysis on the hierarchical $\mathrm{Cu}-\mathrm{Ni} / \mathrm{SiO}_{2}$ catalysts with various $\mathrm{Cu} / \mathrm{Ni}$ ratios and the control sample $\mathrm{IM} \mathrm{Ni} / \mathrm{SiO}_{2}$ at different temperatures ${ }^{[22]}$
的吸附，从而避免 $\mathrm{CH}_{\mathrm{x}, \mathrm{ad}}$ 裂解导致的表面碳沉积。因 此添加碱或碱性氧化物等助剂能够增强载体表面的 碱性和吸附 $\mathrm{CO}_{2}$ 的能力, 改变金属活性中心电子密 度，从而有效提高催化剂的催化活性，同时抑制催 化剂表面碳沉积 ${ }^{[9]}$ 。常见的助剂包括 $\mathrm{K}_{2} \mathrm{O}^{[44-45]}$ 、 $\mathrm{CaO}^{[46]} 、 \mathrm{MgO}^{[47-48]}$ 等碱/碱土金属氧化物, $\mathrm{La}_{2} \mathrm{O}_{3}{ }^{[49]}$ 、 $\mathrm{CeO}_{2}{ }^{[50]}$ 等镧系金属氧化物以及 $\mathrm{V}_{2} \mathrm{O}_{3}{ }^{[51]} 、 \mathrm{Ga}_{2} \mathrm{O}_{3}{ }^{[52]}$ 等 金属氧化物。

$\mathrm{Xu}$ 等 ${ }^{[36]}$ 通过蒸发诱导自组装法制备了一系列 具有不同 $\mathrm{Ca}$ 含量的 $\mathrm{NiO}-\mathrm{CaO}-\mathrm{Al}_{2} \mathrm{O}_{3}$ 三元有序介孔 氧化物, 并研究了 $\mathrm{Ca}$ 含量对 $\mathrm{CO}_{2}$ 吸附能力的影响。 $\mathrm{CO}_{2}$-TPD 脱附曲线(图 4)显示, 在 $170 \sim 215^{\circ} \mathrm{C}$ 和 437 $464^{\circ} \mathrm{C}$ 温度区间，脱附峰强度和面积随着 $\mathrm{Ca}$ 含量的 增加而增强, 峰顶位置也朝高温方向移动, 表明加 入 $\mathrm{Ca}$ 增加了表面碱性活性位点，增强了 $\mathrm{CO}_{2}$ 的吸附 能力。经过 $50 \mathrm{~h}$ 催化后的 TGA 曲线显示, 掺杂 $\mathrm{Ca}$ 后催化剂由碳氧化造成的热失重显著下降，表明添 加 $\mathrm{Ca}$ 元素有助于抑制和消除积碳。他们认为 $\mathrm{Ca}$ 在 抑制积碳中发挥了双重作用: 一方面, 极大降低了 催化剂的酸性，抑制了由表面酸性造成的热解碳; 另一方面, 促进了 $\mathrm{CO}_{2}$ 的化学吸附和活化, 加速了 碳消除反应 $\mathrm{CO}_{2}+\mathrm{C} \rightarrow 2 \mathrm{CO}$ 的进行, 从而有助于消除 催化剂积碳。Pan 等 ${ }^{[22]}$ 研究了 $\mathrm{Ga}_{2} \mathrm{O}_{3}$ 助剂对 $\mathrm{Ni} / \mathrm{SiO}_{2}$ 催化剂性能的影响, 转化率曲线和催化后 TPO 结果 表明, 加入 $\mathrm{Ga}_{2} \mathrm{O}_{3}$ 显著提高了催化剂的转化率和抗 积碳性能。FT-IR 表明加入 $\mathrm{Ga}_{2} \mathrm{O}_{3}$ 助剂不但有助于 提高催化剂对 $\mathrm{CO}_{2}$ 的吸附能力, 而且能够将吸附的 $\mathrm{CO}_{2}$ 活化为碳酸类或碳酸氢类物种, 从而加速了 $\mathrm{CO}_{2}$ 对于沉积碳的消除。

一些助剂的添加还有助于提高金属粒子的分散 度, 起到增强金属-载体相互作用力, 固定金属纳米

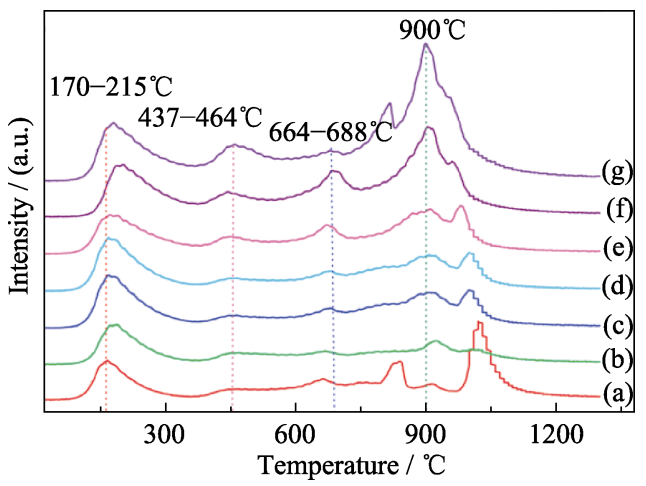

图 4 不同 $\mathrm{Ca}$ 含量的 $\mathrm{M}-5 \mathrm{Nix} \mathrm{Ca}(95-x) \mathrm{Al}$ 在 $600^{\circ} \mathrm{C}$ 㷽烧后的 $\mathrm{CO}_{2}$-TPD 曲线 ${ }^{[36]}$

Fig. $4 \mathrm{CO}_{2}$-TPD profiles of the $\mathrm{M}-5 \mathrm{Ni} x \mathrm{Ca}(95-x) \mathrm{Al}$ materials calcined at $600^{\circ} \mathrm{C}$ with different $\mathrm{Ca}$ contents ${ }^{[36]}$

(a) M-5Ni95Al; (b) M-5Ni1Ca94Al; (c) M-5Ni2Ca93Al; (d) M-5Ni3Ca92Al; (e) M-5Ni5Ca90Al; (f) M-5Ni8Ca87Al; (g) M-5Ni10Ca85Al 
颗粒，阻碍金属粒子的烧结长大等作用 ${ }^{[53-55]}$ 。StaggWilliams 等 ${ }^{[50]}$ 制备了 $\mathrm{Pt} / \mathrm{ZrO}_{2} 、 \mathrm{Pt} / \mathrm{La}-\mathrm{ZrO}_{2}$ 和 $\mathrm{Pt} / \mathrm{Ce}-\mathrm{ZrO}_{2}$, 比较了不同样品用于甲烷干气重整反应的催化性 能、抗积碳性能和热稳定性。结果显示, 掺杂 $\mathrm{La}$ 和 $\mathrm{Ce}$ 组分后样品的热稳定性有了显著提高, 这是由于 掺入的助剂能稳定氧化锆的四方晶相, 增大样品的 表面积并增加金属粒子附近的 $\mathrm{CO}_{2}$ 吸附中心密度, 进而加速 $\mathrm{CO}_{2}$ 的裂解和 $\mathrm{Pt}$ 颗粒上沉积碳的氧化, 从 而增强催化剂的稳定性。另外, 掺入助剂还会抑制 $\mathrm{Pt}$ 颗粒的生长, 从而增加金属的活性位点和金属-载 体界面面积, 这一工作同样对提高镍基催化剂的稳 定性具备参考价值。近期 Margossian 等 ${ }^{[55]}$ 利用 $\left[\left(\mathrm{Ni}\left(\mu^{2}-\mathrm{OCHO}\right)(\mathrm{OCHO})(\text { tmed } a)\right)_{2}\left(\mu^{2}-\mathrm{OH}_{2}\right)\right]$ 前驱体在 $\mathrm{Al}_{2} \mathrm{O}_{3}$ 上生长了尺寸为 $(2 \pm 1) \mathrm{nm}$ 的 $\mathrm{Ni}$ 单质颗粒, 并 通过原位 X 射线吸收近边结构(XANES)等方法研究 了催化剂失活的机理。他们发现 $\mathrm{Ni}$ 单质在反应过程 中向载体晶格内部迁移形成 $\mathrm{NiAl}_{2} \mathrm{O}_{4}$, 使得催化剂活 性在 $20 \mathrm{~h}$ 内下降了 $95 \%$ 。他们通过浸渍将 $\mathrm{Mg}^{2+}$ 离子 导入到了 $\gamma-\mathrm{Al}_{2} \mathrm{O}_{3}$ 载体晶格当中, 掺杂样品在 $20 \mathrm{~h}$ 后 催化活性仅下降了约 40\%(图 5), 表明 $\mathrm{Mg}$ 掺杂的氧 化铝载体可以有效限制 Ni 迁移造成的失活, 增强催 化剂的稳定性。根据 Valentini 等 ${ }^{[51]}$ 研究结果, $V$ 助剂 同样也能在 $\mathrm{Ni} / \gamma-\mathrm{Al}_{2} \mathrm{O}_{3}$ 中起到抑制 $\mathrm{NiAl}_{2} \mathrm{O}_{4}$ 尖晶石相形 成的作用，从而提高 $\mathrm{Ni}$ 金属颗粒的高温稳定性。

\section{4 催化剂结构}

纳米催化剂有几种常见结构, 最常见有核壳(CoreShell)结构、蛋黄-蛋壳(Yolk-Shell)结构、两面神(Janus) 结构和多级结构(Hierarchical structure)等。介孔结构 和 MOF(Metal-Organic Frameworks)结构由于高温 稳定性较差, 在甲烷干气重整催化中的研究比较少 见。其中核壳结构是通过氧化物前驱体水解并沉积

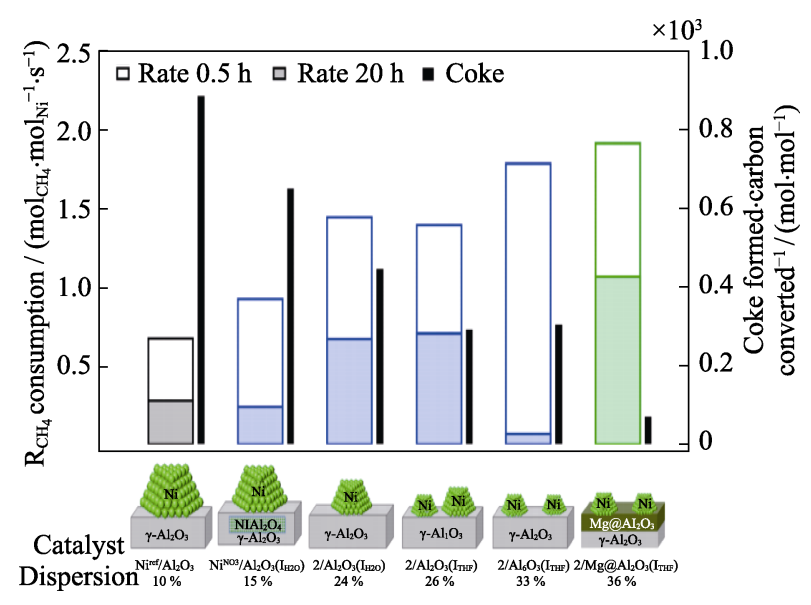

图 5 不同催化剂的甲烷干气重整性能和碳沉积量 ${ }^{[55]}$

Fig. 5 Dry reforming performance and coke formed of different catalysts $^{[55]}$
在金属纳米颗粒周围形成包覆的外壳 ${ }^{[56]}$, 利用 Stober 法可以形成 $\mathrm{SiO}_{2}{ }^{[57]} 、 \mathrm{TiO}_{2}{ }^{\left[{ }^{[8]}\right.} 、 \mathrm{Al}_{2} \mathrm{O}_{3}{ }^{[59]}$ 和 $\mathrm{ZrO}_{2}{ }^{[60]}$ 等一系列金属氧化物核壳结构。由于核壳结构可以 有效防止催化剂活性金属的烧结，同时防止积碳产生， 因此这一特殊结构受到了许多研究者的关注 ${ }^{[18,57,61]}$ 。

本课题组 ${ }^{[61]}$ 通过微乳液法制备了一种核壳结 构催化剂 $\mathrm{Cu}-\mathrm{Ni} @ \mathrm{SiO}_{2}$, 研究了氧化硅外壳对合金 纳米颗粒的生长和稳定性的影响。TEM 显示在未添 加 TEOS 时, 得到的 $\mathrm{Cu}-\mathrm{Ni}$ 合金纳米颗粒尺寸分布 极不均匀, 粒径从 5 至 $50 \mathrm{~nm}$ 不等; 在制备过程中添 加 TEOS 后成功得到 $\mathrm{SiO}_{2}$ 包覆的粒径约为 $12 \mathrm{~nm}$ 的 $\mathrm{Cu}-\mathrm{Ni}$ 单分散纳米颗粒(图 6), 这表明二氧化硅壳层 的存在能够有限限制 $\mathrm{Cu}-\mathrm{Ni}$ 纳米颗粒的长大。此外, 还对比了 $\mathrm{Cu}-\mathrm{Ni} @ \mathrm{SiO}_{2}$ 与传统浸渍法催化剂 $\mathrm{Ni} / \mathrm{SiO}_{2}$ 的催化性能, 后者在 $16 \mathrm{~h}$ 催化中活性迅速下降了 $30 \%$, 而 $\mathrm{Cu}-\mathrm{Ni} @ \mathrm{SiO}_{2}$ 催化剂催化性能仍保持稳定。 从 $\mathrm{Cu}_{1} \mathrm{Ni}_{2.5} @ \mathrm{SiO}_{2}$ 长时间催化后的 TEM 照片可以看 出, 核壳包覆结构的形貌没有发生破坏, 只有少数 金属纳米颗粒由于金属迁移，尺寸发生了略微增长， 表明 $\mathrm{SiO}_{2}$ 外壳能够起到物理隔离的作用, 限制纳米 颗粒核心在高温下的迁移, 抑制催化剂烧结带来的 失活。

Han 等 ${ }^{[29]}$ 使用颈基丙基三甲氧基硅烷对 $\mathrm{SiO}_{2}$ 纳米球进行了表面改性, 并使用超声法将 $\mathrm{Ni}$ 颗粒负 载在 $\mathrm{SiO}_{2}$ 球表面, HAADF-STEM 分析显示硫醇端 基的针定效应使得 $\mathrm{Ni}$ 颗粒均匀分散在 $\mathrm{SiO}_{2}$ 球表面。 他们用 CTAB 模板剂通过 Stober 法在 $\mathrm{SiO}_{2}$ 球表面包 覆了一层多孔氧化硅外壳, 并研究了包覆结构对 $\mathrm{Ni} / \mathrm{SiO}_{2}$ 催化性能的影响(图 7)。催化研究显示, 没有 包覆的催化剂在 $800^{\circ} \mathrm{C}$ 下长时间催化中活性迅速下 降, 且经过长时间催化的样品产生严重的 $\mathrm{Ni}$ 金属烧 结和积碳现象; 包覆了氧化硅外壳后, 催化剂在 $800^{\circ} \mathrm{C}$ 下连续催化 $170 \mathrm{~h}$ 性能仍没有发生改变, 且氧 化硅外壳有效阻碍了 $\mathrm{Ni}$ 金属烧结和碳沉积, 同时外 壳的多孔结构能有效保证气态反应物和产物在催化
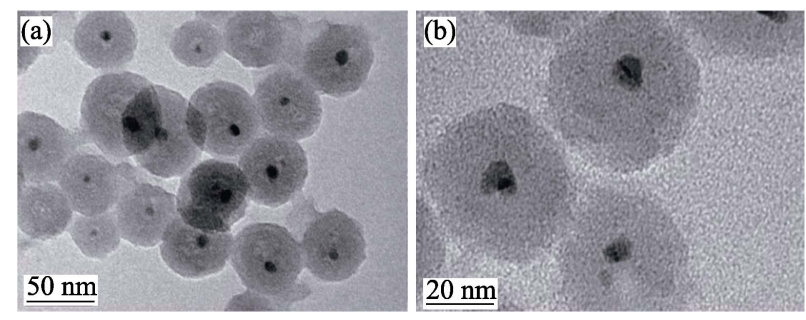

图 6 通过标准路线制备的 $\mathrm{CuNi} @ \mathrm{SiO}_{2}$ 核壳结构催化剂的 TEM 照片 ${ }^{[61]}$

Fig.6 TEM images of $\mathrm{CuNi} @ \mathrm{SiO}_{2}$ core-shell catalysts prepared in the typical synthesis route, a single silica shell contains one core particle $^{[61]}$ 


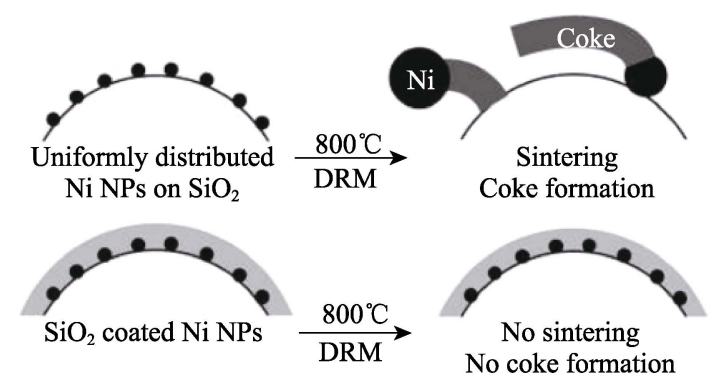

图 7 具备高抗积碳性能的氧化硅包覆结构催化剂用于甲烷 干气重整反应 ${ }^{[29]}$

Fig.7 Silica-coated catalysts with high coke resistance for DRM reaction ${ }^{[29]}$

剂表面的物质转移。最近, Bian 等 ${ }^{[18]}$ 设计了一种二 氧化硅包覆多核纳米棒状结构催化剂 $\mathrm{Ni}-\mathrm{Mg}$ PSNTS@silica, 在 $750^{\circ} \mathrm{C}$ 下长时间催化后仍表现出 较高的稳定性; 未包覆的 Ni-Mg PSNTS 出现了明显 的失活现象, TEM 照片表明失活来自于纳米棒状结 构分解和碳沉积。包覆 $\mathrm{SiO}_{2}$ 后, 介孔氧化硅能够与 层状硅酸盐纳米管的内外表面形成强烈的相互作用, 减小了层状硅酸盐结构在高温下形变, 从而阻碍了 结构内部的破坏; 此外, 由于介孔二氧化硅外壳的 限制效应，以及金属与纳米管载体之间的强相互作 用力, 活性金属烧结和碳沉积得到了有效抑制, 使 得包覆结构显示出高催化活性和稳定性。

除了核壳结构外, 多级结构催化剂同样表现出 了优秀的催化活性和抗积碳性能。多级结构催化剂 一般具备高比表面积和开放空间，使反应气体更容 易与催化剂接触，同时增加了 $\mathrm{Ni}$ 金属的表面分散

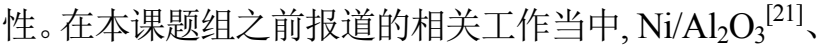
$\mathrm{Cu}-\mathrm{Ni} / \mathrm{SiO}_{2}{ }^{[61]}$ 及 $\mathrm{Ni} / \mathrm{SiO}_{2}{ }^{\left[{ }^{[3]}\right]}$ 催化剂均为多级空心球 结构, 这一特殊结构使催化剂表现出优秀的催化活 性和稳定性。 $\mathrm{Du}$ 等 ${ }^{[62]}$ 报道了一种具有多级结构的 HT-NiMgAl 甲烷干气重整催化剂(图 8), 这一多孔 多级结构有效增大了催化剂比表面积, 从而有助于 提高 $\mathrm{Ni}$ 颗粒的分散性; 同时，催化剂表面的多孔多 级结构纳米片具备限制效应，能够有效抑制碳沉积 及 $\mathrm{Ni}$ 金属颗粒的烧结。 $\mathrm{Li}$ 等 ${ }^{[63]}$ 制备了一系列 $\mathrm{Ni} / \mathrm{ZrO}_{2}$ 催化剂并对比了它们的甲烷干气重整催化 性能。与其它催化剂相比, 具备大丽花状多级结构 的 $\mathrm{Ni} / \mathrm{ZrO}_{2}$ 催化剂表现出更高的催化活性和选择性, 被归因于特殊的载体多级结构所导致的 $\mathrm{Ni}$ 颗粒的 高分散性、 $\mathrm{NiO}$ 的易还原性、更高的晶格比率和较 强的碱性。此外, 与常规 $\mathrm{ZrO}_{2}$ 纳米颗粒负载的催化 剂相比，大丽花状 $\mathrm{ZrO}_{2}$ 负载的镍基催化剂同样表现 出更高的催化稳定性和更优秀的抗积碳性能。

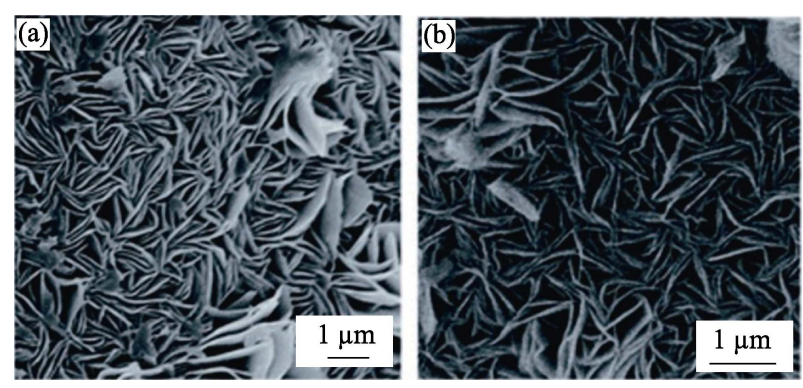

图 8 (a)㷽烧前和(b)还原后 HT-NiMgAl 的 SEM 照片 ${ }^{[62]}$

Fig. 8 SEM images of the HT-NiMgAl (a) before calcination and (b) after reduction ${ }^{[62]}$

\section{5 等离子体处理}

近期，通过等离子体法制备具有高积碳性能的 镍基催化剂受到了广泛重视 ${ }^{[64-65]}$ 。等离子体中的活 性电子、离子和自由基能够调控金属颗粒尺寸、形 貌和金属-载体相互作用力，因此等离子体处理可 以增加活性金属组分的分散性和表面浓度，进而显 著提高催化剂的活性和抗积碳性能 ${ }^{[66-67]}$ 。常见的等 离子体处理方法包括辉光放电、电晕放电、微波放 电和等离子体喷涂、介质阻挡放电等。Liu 等 ${ }^{[65]}$ 系 统性地研究了通过高温和低温等离子体技术制备催 化剂的方法，其中三种常见的用于制备催化剂的等 离子体(辉光放电，微波等离子体和等离子喷涂)电 极装置示意图如图 9 所示。他们总结认为, 与常规 催化剂相比，通过等离子体技术制备的催化剂主要 具备以下几点优势：(1)能够得到具备高比表面积的 超分散催化剂; (2)样品中组分的均匀分布, 有助于 降低催化剂中毒的敏感性; (3)增强了催化剂的活 性、选择性和使用寿命; (4)缩短了催化剂制备时间; (5)样品的分散度和组分可以通过调节等离子体化 学参数的变化来控制等。他们还通过辉光放电等离 子体法制备了甲烷干气重整催化剂 $\mathrm{Ni} / \mathrm{Al}_{2} \mathrm{O}_{3}{ }^{[68]}$ 。与 此前报道的工作相比，该催化剂表现出了更高的低 温催化活性，在 $650^{\circ} \mathrm{C}$ 下 $\mathrm{CH}_{4}$ 转化率提高了约 $20 \%$ 。 此外，等离子体法处理的催化剂表现出更强的抗积 碳性能，镍金属颗粒在长时间催化后仍保持高度分 散性。

Liu 等 ${ }^{[69]}$ 通过等离子体辅助煅烧和还原制备了 $\mathrm{Ni} / \mathrm{SiO}_{2}$ 催化剂, 他们以大气高频冷等离子射流对前 驱体进行了 $10 \mathrm{~min}$ 的活化, 取代了传统制备过程中 的长时间高温煅烧和还原。经过等离子体活化处理 的催化剂具备更小的 $\mathrm{Ni}$ 颗粒尺寸、高分散性和更大 的比表面积，并且没有发生 $\mathrm{Ni}$ 金属颗粒烧结或团聚 的现象(图 10); 经过等离子体处理的催化剂在 $800^{\circ} \mathrm{C}$ 下 $\mathrm{CO}_{2}$ 转化率高达 $93 \%$ ，与传统方法制备的催化剂 相比转化率提高了 $20 \%$ 。 $\mathrm{Zhu}$ 等 ${ }^{[64]}$ 对比了通过 


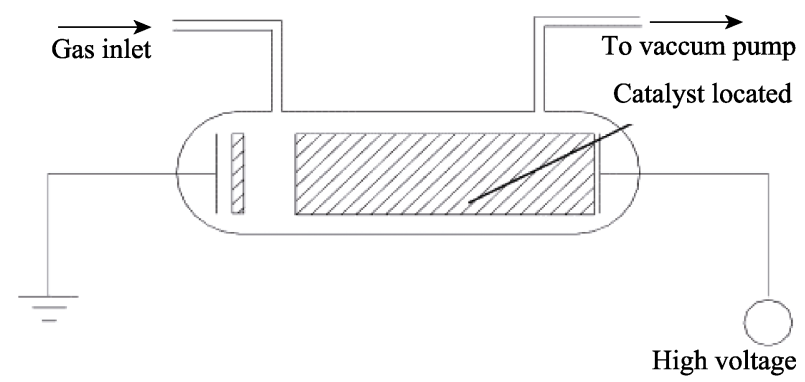

(a)

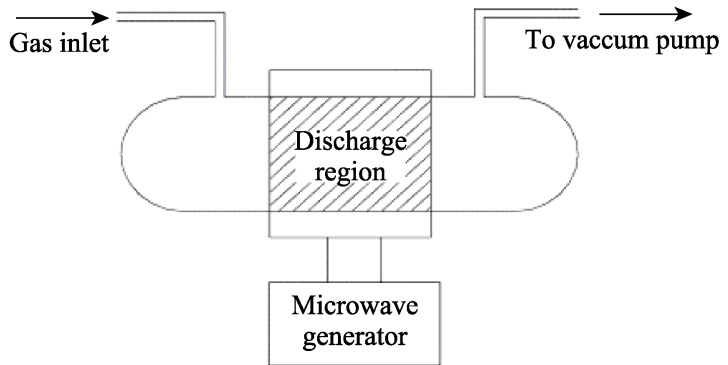

(b)

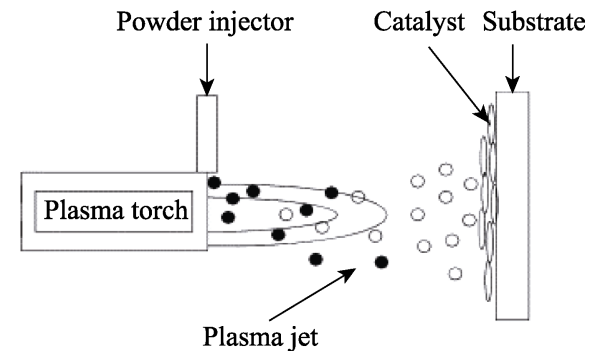

(c)

图 9 用于制备催化剂的放电电极结构示意图 ${ }^{[65]}$

Fig. 9 Schematically representatives of electrode configurations of discharge phenomena applied for catalyst preparation ${ }^{[65]}$ (a) Glow discharge; (b) Microwave plasma; (c) Plasma spraying (a)
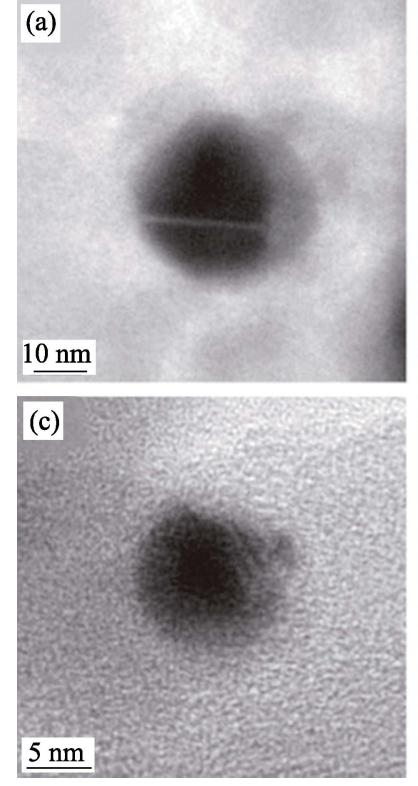
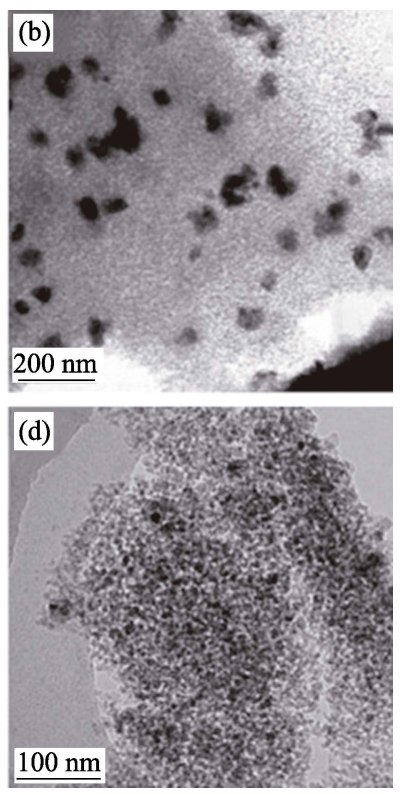

图 10 通过两种路线制备的(a) (b) 常规催化剂和(c) (d)等 离子体法制备的新型催化剂的 TEM 照片 ${ }^{[69]}$

Fig. 10 TEM images of $\mathrm{Ni} / \mathrm{SiO}_{2}$ catalyst samples prepared by two methods $(a, b)$ the conventional catalyst and $(c, d)$ the novel catalyst prepared by plasma jet ${ }^{[69]}$
辉光放电等离子体和常规热处理制备的 $\mathrm{Ni} / \mathrm{Al}_{2} \mathrm{O}_{3}$ 催 化剂的性能, $\mathrm{CO}$ 吸附的傅里叶漫反射红外光谱 (DRIFTS)表明等离子体处理能够增加 $\mathrm{Ni}$ 颗粒的密 堆积面，进而提高催化剂的抗积碳性能。

\subsection{DFT 计算}

要从根本上解决镍基催化剂所存在的问题, 需 要深入且清晰地揭示催化反应机理和积碳过程, 在 原子尺度上建立起结构与催化活性的关系, 从反应 动力学和反应途径角度研究具有高活性和稳定性的 镍基催化剂对干气重整反应的影响, 更合理地设计 和开发出性能最优的催化剂体系。因此许多研究者 通过密度泛函理论(Density Functional Theory, DFT) 等计算方法对 $\mathrm{Ni}$ 基催化剂的反应动力学、催化机理 和积碳机理等进行了理论研究, 以期通过实验和计 算结果优化镍基催化剂的催化性能和抗积碳能力。

Wang 等 ${ }^{[70]}$ 综合了反应物吸附解离和脱附过程, 通过计算推测了 $\mathrm{Ni}(111)$ 晶面上甲烷干气重整反应 的过程: 首先 $\mathrm{CH}_{4}$ 逐步活化 $\left(\mathrm{CH}_{4} \rightarrow \mathrm{CH}_{3} \rightarrow \mathrm{CH}_{2} \rightarrow\right.$ $\mathrm{CH})$ 解离生成表面 $\mathrm{CH}$, 同时 $\mathrm{CO}_{2}$ 活化 $\left(\mathrm{CO}_{2} \rightarrow \mathrm{CO}+\right.$ $\mathrm{O})$ 解离出 $\mathrm{CO}$ 和 $\mathrm{O}$; 随后 $\mathrm{CH}$ 被 $\mathrm{O}$ 氧化生成 $\mathrm{CHO}(\mathrm{H}+$ $\mathrm{O} \rightarrow \mathrm{CHO})$; 最后 $\mathrm{CHO}$ 再解离成为 $\mathrm{CO}$ 和 $\mathrm{H}(\mathrm{CHO} \rightarrow$ $\mathrm{CO}+\mathrm{H})$ 。同时他们指出, $\mathrm{CH}_{4}$ 活化解离为 $\mathrm{CH}_{3}$ 和 $\mathrm{H}$ 是 $\mathrm{Ni}(111)$ 晶面上甲烷干重整反应的决速步骤; $\mathrm{CH}$ 更倾向于被氧化生成 $\mathrm{CHO}$ 而非进一步解离成为 $\mathrm{C}$ 和 $\mathrm{H}$, 因此 $\mathrm{Ni}$ 表面的积碳来源主要是 $\mathrm{CO}_{2}$ 的解离。 Wang 等 ${ }^{\left[{ }^{[1]}\right.}$ 也通过 DFT 方法和微观动力学模型研究 了在 $\mathrm{Ni}(111) 、 \mathrm{Ni}(211) 、 \mathrm{Ni}_{3} \mathrm{C}(001)$ 和 $\mathrm{Ni}_{3} \mathrm{C}(111)$ 晶面 上的甲烷干气重整反应，进而揭示反应活性位点和 失活机理。计算表明, 在不同晶面上重整反应的催 化活性顺序为 $\mathrm{Ni}(111)>\mathrm{Ni}_{3} \mathrm{C}(001)>\mathrm{Ni}(211)>\mathrm{Ni}_{3} \mathrm{C}(111)$, 进而可以得出结论：(1) 光滑的表面 $\mathrm{Ni}(111)$ 和 $\mathrm{Ni}_{3} \mathrm{C}(001)$ 比阶梯状表面 $\mathrm{Ni}(211)$ 和 $\mathrm{Ni}_{3} \mathrm{C}(111)$ 具备更 高的干气重整催化活性; (2)在 $\mathrm{Ni}$ 金属单质晶面上的 催化活性高于碳化镍晶面, 因此积碳造成的 $\mathrm{Ni}$ 单质 相变生成碳化镍会导致催化活性的下降(图 11)。他 们同样对于积碳进行了研究, 结果表明在 $\mathrm{Ni}(111)$ 平 面上几乎不会产生积碳，而在阶梯状的镍单质和碳 化镍晶面上很容易发生积碳现象, 因此晶面不仅具 备最高的催化活性，还具备最强的抗积碳性能。他 们认为对于活性位点和积碳的分析计算结果不仅可 以对甲烷干气重整催化剂的设计提供指导, 还同样 适用于其他受积碳问题影响的催化体系，如固体燃 料电池和甲烷水蒸气重整等。

由于研究者所使用的理论模型、方法以及参数 等的差异, 计算结果不尽相同。而实验中由于多种 


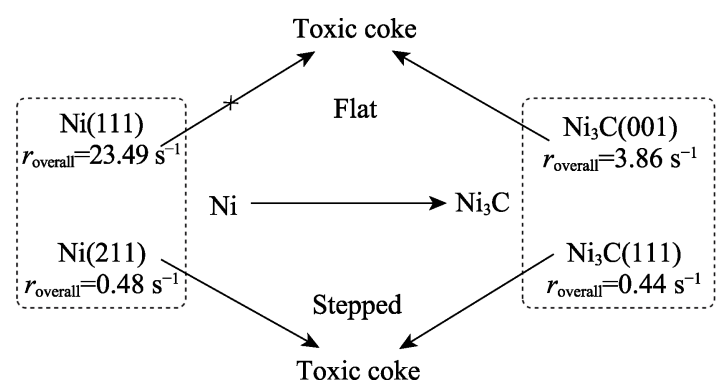

图 11 相变及积碳造成的失活示意图 ${ }^{[71]}$

Fig. 11 Deactivation scheme to show schematically the phase transformation and coke formation ${ }^{[71]}$

$\mathrm{Ni}(111)$ and $\mathrm{Ni}(211)$ stand for the clean nickel metal flat and stepped surfaces respectively, and $\mathrm{Ni}_{3} \mathrm{C}(001)$ and $\mathrm{Ni}_{3} \mathrm{C}(111)$ are the flat and stepped nickel carbide. Flat surfaces are marked in blue, while stepped surfaces are in red. Toxic coke is the more stable and high toxicity carbon atom which will result in the deactivation of the nickel catalyst. The corresponding overall reaction rates are marked below the different structures. The arrow with $\mathrm{X}$ means this step is not likely to occur, while arrow without $\mathrm{X}$ means this step could happen

因素的综合影响导致实验与模拟计算的结果存在一 些差异。但是随着计算模型的进一步完善以及计算 能力的不断强大，包括高通量计算能力的不断进步， 理论计算对实验现象的解释将越来越合理, 对实验 方向指导以及实验结果的预测也将越来越准确。模 拟计算将成为催化科学不可缺少的一部分。

\section{3 总结与展望}

甲烷干气重整反应是一个复杂的过程, 催化剂 的尺寸、形状、结构和表面组分都会影响催化活性 和稳定性。目前关于提高负载型镍基催化剂性能的 主要方法和思路有:

1) 提高金属-载体相互作用力, 抑制 $\mathrm{Ni}$ 颗粒在 载体表面的移动生长;

2) 将 $\mathrm{Ni}$ 合金化, 利用双金属协同效应提高催化 活性和稳定性;

3) 添加合适的助剂, 改变催化剂表面酸碱性和 金属稳定性;

4) 设计合适的催化剂结构, 通过限制效应防止 $\mathrm{Ni}$ 颗粒迁移烧结;

5) 对催化剂前驱体进行等离子体处理, 从而提 高金属的分散性和催化剂抗积碳性能;

6) 通过 DFT 计算研究催化和积碳机理, 指导 和优化催化剂设计。

其中活性金属 $\mathrm{Ni}$ 的尺寸是最重要的影响因素, 对于催化剂组分、结构和形貌的研究实际上是对尺 寸控制的研究, 如果在重整反应过程中 $\mathrm{Ni}$ 颗粒尺寸 可以被限制或保持在若干纳米 $(<9 \mathrm{~nm})$, 那么就可以 完全抑制积碳形成 ${ }^{[20]}$ 。除了前文详细介绍的方法外,
研究人员还从表面氧化还原反应 ${ }^{[72]}$ 、表面氧物种 ${ }^{[73]}$ 和羟基 ${ }^{[74]}$ 等方面进行催化剂的设计和制备, 也取得 了有效的结果。

近几年, 新兴的单原子催化剂在甲烷催化转化 领域获得了突破性进展, 然而单原子/亚纳米级别的 镍基催化剂在甲烷干气重整方面的应用却仍未见报 道。本课题组使用原子层沉积技术(Atomic Layer Deposition, ALD)进行了甲烷干气镍基重整单原子 催化剂技术初步探索, 相信经过研究人员的共同努 力, 单原子催化剂在甲烷干气重整催化和机理探究 上会获得进展和突破。此外，原位 TEM、XRD、FT-IR 和 XPS 等原位催化表征技术的进步也为甲烷干气 重整催化剂的原理研究和结构组分设计提供了更为

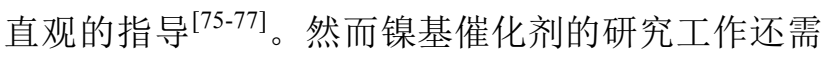
要面对一些权衡的问题。一方面, 在各项参数的调控 上有时候要面临一定的选择和平衡: 研究大多集中 于制备高温稳定的催化剂, 但催化剂的结构稳定性 与催化过程中的质流动力学之间存在一定的矛盾; 助剂能够提升催化剂性能, 但添加助剂又可能部分 涂覆金属催化剂的活性位点; 对于镍基等非贵金属 的不断活化本身也增加了运行成本等。另一方面, 在 镍基催化剂的表征上仍存在一定困难：镍基催化剂 存在磁性，这在进行电镜表征尤其是高分辨表征时 受到一些限制; 双金属催化剂中双金属与合金纳米 颗粒往往共存, 这对催化剂构效关系的解析也增加 了很多困难，通常需要用更高分辨率的电镜来表征; 由于干气重整反应的高温环境, 原位 TEM 等一些原 位表征手段尚不能直接应用于甲烷干气重整反应过 程的探究等。这些问题需要通过催化剂制备和研究 表征方法的不断发展来得到解决。

\section{参考文献:}

[1] TOLLEFSON J. World looks ahead post-Copenhagen. Nature, 2009, 462(7276): 966-967.

[2] TOLLEFSON J. Copenhagen: the scientists' view. Nature, 2009, 462(7274): 714.

[3] WANG W, WANG S P, MA X B, et al. Recent advances in catalytic hydrogenation of carbon dioxide. Chem. Soc. Rev., 2011, 40(7): 3703-3727.

[4] TOLLEFSON J. World's carbon emissions set to spike by $2 \%$ in 2017. Nature News, 2017, 551(7680): 283.

[5] SONG C S. Global challenges and strategies for control, conversion and utilization of $\mathrm{CO}_{2}$ for sustainable development involving energy, catalysis, adsorption and chemical processing. Catal. Today, 2006, 115(1-4): 2-32.

[6] MELIKOGLU M. Shale gas: analysis of its role in the global energy market. Renew Sust. Energ. Rev., 2014, 37: 460-468.

[7] BERNARD S, HORSFIELD B. Thermal maturation of gas shale systems. Annu. Rev. Earth Planet. Sci., 2014, 42(1): 635-651.

[8] WANG N, YU X, SHEN K, et al. Synthesis, characterization and 
catalytic performance of $\mathrm{MgO}$-coated $\mathrm{Ni} / \mathrm{SBA}-15$ catalysts for methane dry reforming to syngas and hydrogen. Int. J. Hydrogen Energy, 2013, 38(23): 9718-9731.

[9] SIBUDJING K, YASOTHA K, JUN N, et al. Progress in synthesis of highly active and stable nickel-based catalysts for carbon dioxide reforming of methane. ChemSusChem, 2015, 8(21): 3556-3575.

[10] ASSABUMRUNGRAT S, CHAROENSERI S, LAOSIRIPOJANA $\mathrm{N}$, et al. Effect of oxygen addition on catalytic performance of $\mathrm{Ni} / \mathrm{SiO} \cdot \mathrm{MgO}$ toward carbon dioxide reforming of methane under periodic operation. Int. J. Hydrogen Energy, 2009, 34(15): 6211-6220.

[11] KIM H Y, PARK J N, HENKELMAN G, et al. Design of a highly nanodispersed $\mathrm{Pd}-\mathrm{MgO} / \mathrm{SiO}_{2}$ composite catalyst with multifunctional activity for $\mathrm{CH}_{4}$ reforming. ChemSusChem, 2012, 5(8): 1474-1481.

[12] FAN M S, ABDULLAH A Z, BHATIA S. Catalytic technology for carbon dioxide reforming of methane to synthesis gas. ChemCatChem, 2009, 1(2): 192-208.

[13] WANG S, LU G Q, MILLAR G J. Carbon dioxide reforming of methane to produce synthesis gas over metal-supported catalysts: state of the art. Energy \& Fuels, 1996, 10(4): 896-904.

[14] ZHANG J, WANG H, DALAI A K. Development of stable bimetallic catalysts for carbon dioxide reforming of methane. $J$. Catal., 2007, 249(2): 300-310.

[15] TOPALIDIS A, PETRAKIS D, LADAVOS A, et al. A kinetic study of methane and carbon dioxide interconversion over $0.5 \%$ $\mathrm{Pt} / \mathrm{SrTiO}_{3}$ catalysts. Catal. Today, 2007, 127(1): 238-245.

[16] PAKHARE D, SPIVEY J. A review of dry $\left(\mathrm{CO}_{2}\right)$ reforming of methane over noble metal catalysts. Chem. Soc. Rev., 2014, 43(22): 7813-7837.

[17] BRADFORD M C J, VANNICE M A. $\mathrm{CO}_{2}$ reforming of $\mathrm{CH}_{4}$ over supported Pt catalysts. J. Catal., 1998, 173(1): 157-171.

[18] BIAN Z, SURYAWINATA I Y, KAWI S. Highly carbon resistant multicore-shell catalyst derived from $\mathrm{Ni}-\mathrm{Mg}$ phyllosilicate nanotubes@silica for dry reforming of methane. Appl. Catal., B, 2016, 195: $1-8$.

[19] TSYGANOK A I, TSUNODA T, HAMAKAWA S, et al. Dry reforming of methane over catalysts derived from nickel-containing Mg-Al layered double hydroxides. J. Catal., 2003, 213(2): 191203.

[20] LIU C J, YE J, JIANG J, et al. Progresses in the preparation of coke resistant Ni-based catalyst for steam and $\mathrm{CO}_{2}$ reforming of methane. ChemSusChem, 2015, 3(3): 529-541.

[21] ZHANG Q, WU T, ZHANG P, et al. Facile synthesis of hollow hierarchical $\mathrm{Ni} / \mathrm{g}-\mathrm{Al}_{2} \mathrm{O}_{3}$ nanocomposites for methane dry reforming catalysis. RSC Adv, 2014, 4(93): 51184-51193.

[22] WU T, ZHANG Q, CAI W, et al. Phyllosilicate evolved hierarchical Ni- and $\mathrm{Cu}-\mathrm{Ni} / \mathrm{SiO}_{2}$ nanocomposites for methane dry reforming catalysis. Appl. Catal. A, 2015, 503: 94-102.

[23] DAZA C E, GALLEGO J, MONDRAG N F, et al. High stability of Ce-promoted Ni/Mg-Al catalysts derived from hydrotalcites in dry reforming of methane. Fuel, 2010, 89(3): 592-603.

[24] FERREIRA-APARICIO P, FERNANDEZ-GARCIA M, GUERRERO-RUIZ A, et al. Evaluation of the role of the metal-support interfacial centers in the dry reforming of methane on alumina- supported rhodium catalysts. J. Catal., 2000, 190(2): 296-308.

[25] CHETTAPONGSAPHAN C, CHAROJROCHKUL S, ASSABUMRUNGRAT S, et al. Catalytic $\mathrm{H}_{2} \mathrm{O}$ and $\mathrm{CO}_{2}$ reforming of $\mathrm{CH}_{4}$ over perovskite-based $\mathrm{La}_{0.8} \mathrm{Sr}_{0.2} \mathrm{Cr}_{0.9} \mathrm{Ni}_{0.1} \mathrm{O}_{3}$ : effects of pre-treatment and co-reactant $/ \mathrm{CH}_{4}$ on its reforming characteristics. Appl. Catal., 2010, 386(1/2): 194-200.

[26] GALLEGO G S, BATIOT-DUPEYRAT C, BARRAULT J, et al.
Dry reforming of methane over $\mathrm{LaNi}_{1-y} \mathrm{~B}_{y} \mathrm{O}_{3 \pm \delta}(\mathrm{B}=\mathrm{Mg}, \mathrm{Co})$ perovskites used as catalyst precursor. Appl. Catal. A, 2008, 334(1/2): 251-258.

[27] GARC A-DI GUEZ M, PIETA I, HERRERA M, et al. Transient study of the dry reforming of methane over Pt supported on different $\gamma-\mathrm{Al}_{2} \mathrm{O}_{3}$. Catal. Today, 2010, 149(3/4): 380-387.

[28] LUCR DIO A F, ASSAF J M, ASSAF E M. Methane conversion reactions on Ni catalysts promoted with $\mathrm{Rh}$ : influence of support. Appl. Catal. A, 2011, 400(1/2): 156-165.

[29] HAN J W, KIM C, PARK J S, et al. Highly coke-resistant Ni nanoparticle catalysts with minimal sintering in dry reforming of methane. ChemSusChem, 2014, 7(2): 451-456.

[30] ALIEH, KHALESI, HAMID, et al. Effects of lanthanum substitution by strontium and calcium in La-Ni-Al perovskite oxides in dry reforming of methane. Chin. J. Catal., 2008, 29(10): 960-968.

[31] LI X, LI D, TIAN H, et al. Dry reforming of methane over $\mathrm{Ni} / \mathrm{La}_{2} \mathrm{O}_{3}$ nanorod catalysts with stabilized Ni nanoparticles. Appl. Catal. B, 2017, 202: 683-694.

[32] LUNA A E C, IRIARTE M E. Carbon dioxide reforming of methane over a metal modified $\mathrm{Ni}-\mathrm{Al}_{2} \mathrm{O}_{3}$ catalyst. Appl. Catal. A, 2008, 343(1): 10-15.

[33] XU Z, ZHEN M, BI Y, et al. Catalytic properties of Ni modified hexaaluminates $\mathrm{LaNi}_{y} \mathrm{Al}_{12-y} \mathrm{O}_{19-\delta}$ for $\mathrm{CO}_{2}$ reforming of methane to synthesis gas. Appl. Catal., A, 2000, 198(1): 267-723.

[34] $\mathrm{HU}$ Y H. Solid-solution catalysts for $\mathrm{CO}_{2}$ reforming of methane. Catal. Today, 2009, 148(3): 206-211.

[35] JAFARBEGLOO M, TARLANI A, MESBAH A W, et al. $\mathrm{NiO}-\mathrm{MgO}$ Solid solution prepared by Sol-Gel method as precursor for $\mathrm{Ni} / \mathrm{MgO}$ methane dry reforming catalyst: effect of calcination temperature on catalytic performance. Catal. Lett., 2016, 146(1): $238-248$.

[36] XU L, SONG H, CHOU L. One-pot synthesis of ordered mesoporous $\mathrm{NiO}-\mathrm{CaO}-\mathrm{Al}_{2} \mathrm{O}_{3}$ composite oxides for catalyzing $\mathrm{CO}_{2}$ reforming of $\mathrm{CH}_{4}$. ACS Catalysis, 2012, 2(7): 1331-1342.

[37] ZUBENKO D, SINGH S, ROSEN B A. Exsolution of Re-alloy catalysts with enhanced stability for methane dry reforming. Appl. Catal. B, 2017, 209: 711-719.

[38] LI Y, ZHANG Q, LIU J, et al. Corona shaped $\mathrm{SiO}_{2}$ supported $\mathrm{Ni}$ nanoparticles for methane dry reforming catalysis. J. Chin. Ceram. Soc., 2015, 43(7): 911-918.

[39] BIAN Z, DAS S, WAI M H, et al. A review on bimetallic Ni-based catalysts for $\mathrm{CO}_{2}$ reforming of methane. ChemPhysChem A, 2017, 18(22): $3117-3134$

[40] TU W, GHOUSSOUB M, SINGH C V, et al. Consequences of surface oxophilicity of $\mathrm{Ni}, \mathrm{Ni}-\mathrm{Co}$, and Co clusters on methane activation. J. Am. Chem. Soc., 2017, 139(20): 6928-6945.

[41] CHEN Y G, YAMAZAKI O, TOMISHIGE K, et al. Noble metal promoted $\mathrm{Ni}_{0.03} \mathrm{Mg}_{0.97} \mathrm{O}$ solid solution catalysts for the reforming of $\mathrm{CH}_{4}$ with $\mathrm{CO}_{2}$. Catal. Lett., 1996, 39(1/2): 91-95.

[42] WU H, PANTALEO G, PAROLA V L, et al. Bi- and trimetallic Ni catalysts over $\mathrm{Al}_{2} \mathrm{O}_{3}$ and $\mathrm{Al}_{2} \mathrm{O}_{3}-\mathrm{MO}_{x}(\mathrm{M}=\mathrm{Ce}$ or $\mathrm{Mg})$ oxides for methane dry reforming: Au and Pt additive effects. Appl. Catal. B, 2014, 156-157(2): 350-361.

[43] HORIUCHI T, SAKUMA K, FUKUI T, et al. Suppression of carbon deposition in the $\mathrm{CO}_{2}$-reforming of $\mathrm{CH}_{4}$ by adding basic metal oxides to a Ni/ $/ \mathrm{Al}_{2} \mathrm{O}_{3}$ catalyst. Appl. Catal. A, 1996, 144(1/2): 111- 120.

[44] OSAKI T, MORI T. Role of potassium in carbon-free $\mathrm{CO}_{2}$ reforming of methane on K-promoted $\mathrm{Ni} / \mathrm{Al}_{2} \mathrm{O}_{3}$ catalysts. J. Catal., 2001, 204(1): 89-97.

[45] FRUSTERI F, SPADARO L, ARENA F, et al. TEM evidence for factors affecting the genesis of carbon species on bare and K-promoted $\mathrm{Ni} / \mathrm{MgO}$ catalysts during the dry reforming of methane. Carbon, 2002, 40(7): 1063-1070. 
[46] DIAS J A C, ASSAF J M. Influence of calcium content in $\mathrm{Ni} / \mathrm{CaO} /$ $\gamma-\mathrm{Al}_{2} \mathrm{O}_{3}$ catalysts for $\mathrm{CO}_{2}$-reforming of methane. Catal. Today, 2003, 85(1): 59-68.

[47] KOO K Y, ROH H S, YU T S, et al. Coke study on MgO-promoted $\mathrm{Ni} / \mathrm{Al}_{2} \mathrm{O}_{3}$ catalyst in combined $\mathrm{H}_{2} \mathrm{O}$ and $\mathrm{CO}_{2}$ reforming of methane for gas to liquid (GTL) process. Appl. Catal. A, 2008, 340(2): 183190.

[48] KATHIRASER Y, THITSARTARN W, SUTTHIUMPORN K, et al. Inverse $\mathrm{NiAl}_{2} \mathrm{O}_{4}$ on $\mathrm{LaAlO}_{3}-\mathrm{Al}_{2} \mathrm{O}_{3}$ : unique catalytic structure for stable $\mathrm{CO}_{2}$ reforming of methane. J. Phys. Chem. C, 2013, 117(16): $8120-8130$.

[49] SUTTHIUMPORN K, KAWI S. Promotional effect of alkaline earth over Ni- $\mathrm{La}_{2} \mathrm{O}_{3}$ catalyst for $\mathrm{CO}_{2}$ reforming of $\mathrm{CH}_{4}$ : role of surface oxygen species on $\mathrm{H}_{2}$ production and carbon suppression. Int. J. Hydrogen Energy, 2011, 36(22): 14435-14446.

[50] STAGG-WILLIAMS S M, FENDLEY G, RESASCO D E, et al. $\mathrm{CO}_{2}$ reforming of $\mathrm{CH}_{4}$ over $\mathrm{Pt} / \mathrm{ZrO}_{2}$ catalysts promoted with $\mathrm{La}$ and Ce oxides. J. Catal., 2000, 194(2): 240-249.

[51] VALENTINI A, PROBST L F D, LISBOA-FILHO P N, et al. Role of vanadium in $\mathrm{Ni}: \mathrm{Al}_{2} \mathrm{O}_{3}$ catalysts for carbon dioxide reforming of methane. Appl. Catal. A, 2003, 255(2): 211-220.

[52] PAN Y, KUAI P, LIU Y, et al. Promotion effects of $\mathrm{Ga}_{2} \mathrm{O}_{3}$ on $\mathrm{CO}_{2}$ adsorption and conversion over a $\mathrm{SiO}_{2}$-supported $\mathrm{Ni}$ catalyst. Energy Environ. Sci., 2010, 3(9): 1322-1325.

[53] HOU Z, YOKOTA O, TANAKA T, et al. Characterization of Ca-promoted $\mathrm{Ni} / \alpha-\mathrm{Al}_{2} \mathrm{O}_{3}$ catalyst for $\mathrm{CH}_{4}$ reforming with $\mathrm{CO}_{2}$. Appl. Catal. A, 2003, 253(2): 381-387.

[54] GUO J, LOU H, ZHAO H, et al. Dry reforming of methane over nickel catalysts supported on magnesium aluminate spinels. Appl. Catal. A, 2004, 273(1): 75-82.

[55] MARGOSSIAN T, LARMIER K, KIM S M, et al. Molecularlytailored nickel precursor and support yield a stable methane dry reforming catalyst with superior metal utilization. J. Am. Chem. Soc., 2017, 139(20): 6919-6927.

[56] LIU S, BAI S Q, ZHENG Y, et al. Composite metal-oxide nanocatalysts. Chem CatChem, 2012, 4(10): 1462-1484.

[57] ZHANG J, LI F. Coke-resistant $\mathrm{Ni} @ \mathrm{SiO}_{2}$ catalyst for dry reforming of methane. Appl. Catal. B, 2015, s176-177: 513-521.

[58] KIM D H, KIM S Y, HAN S W, et al. The catalytic stability of $\mathrm{TiO}_{2}$-shell/Ni-core catalysts for $\mathrm{CO}_{2}$ reforming of $\mathrm{CH}_{4}$. Appl. Catal. A, 2015, 495: 184-191.

[59] BAKTASH E, LITTLEWOOD P, SCHOM CKER R, et al. Alumina coated nickel nanoparticles as a highly active catalyst for dry reforming of methane. Appl. Catal. B, 2015, 179: 122-127.

[60] LIM Z Y, WU C, WANG W G, et al. Porosity effect on $\mathrm{ZrO}_{2}$ hollow shells and hydrothermal stability for catalytic steam reforming of methane. Journal of Materials Chemistry A, 2015, 4(1): 153-159.

[61] WU T, CAI W, ZHANG P, et al. Cu-Ni@ $\mathrm{SiO}_{2}$ alloy nanocomposites for methane dry reforming catalysis. RSC Adv., 2013, 3(46): 23976-23979.
[62] DU X, ZHANG D, SHI L, et al. Coke- and sintering-resistant monolithic catalysts derived from in situ supported hydrotalcite-like films on $\mathrm{Al}$ wires for dry reforming of methane. Nanoscale, 2013, 5(7): 2659-2663

[63] LI W, ZHAO Z, JIAO Y. Dry reforming of methane towards CO-rich hydrogen production over robust supported $\mathrm{Ni}$ catalyst on hierarchically structured monoclinic zirconia nanosheets. Int. J. Hydrogen Energy, 2016, 41(40): 17907-17921.

[64] ZHU X, HUO P, ZHANG Y P, et al. Structure and reactivity of plasma treated $\mathrm{Ni} / \mathrm{Al}_{2} \mathrm{O}_{3}$ catalyst for $\mathrm{CO}_{2}$ reforming of methane. Appl. Catal. B, 2008, 81(1/2): 132-140.

[65] LIU C J, VISSOKOV G P, JANG W L. Catalyst preparation using plasma technologies. Catal. Today, 2002, 72(3/4): 173-184.

[66] LIU C J. Plasma application for more environmentally friendly catalyst preparation. Pure Appl. Chem., 2006, 78(6): 1227-1238.

[67] HONG J, CHU W, CHERNAVSKII P A, et al. Cobalt species and cobalt-support interaction in glow discharge plasma-assisted FischerTropsch catalysts. J. Catal., 2010, 273(1): 9-17.

[68] CHENG D G, ZHU X, BEN Y, et al. Carbon dioxide reforming of methane over $\mathrm{Ni} / \mathrm{Al}_{2} \mathrm{O}_{3}$ treated with glow discharge plasma. Catal. Today, 2006, 115(1-4): 205-210.

[69] LIU G, LI Y, CHU W, et al. Plasma-assisted preparation of $\mathrm{Ni} / \mathrm{SiO}_{2}$ catalyst using atmospheric high frequency cold plasma jet. Catal. Commun., 2008, 9(6): 1087-1091.

[70] WANG S G, CAO D B, LI Y W, et al. $\mathrm{CO}_{2}$ reforming of $\mathrm{CH}_{4}$ on $\mathrm{Ni}$ (111): a density functional theory calculation. J. Phys. Chem. B, 2006, 110(20): 9976-9983.

[71] WANG Z, CAO X M, ZHU J, et al. Activity and coke formation of nickel and nickel carbide in dry reforming: a deactivation scheme from density functional theory. $J$. Catal., 2014, 311(3): 469-480.

[72] AW M S, ZORKO M, DJINOVIĆ P, et al. Insights into durable NiCo catalysts on $\beta$-SiC/CeZrO 2 and $\gamma-\mathrm{Al}_{2} \mathrm{O}_{3} / \mathrm{CeZrO}_{2}$ advanced supports prepared from facile methods for $\mathrm{CH}_{4}-\mathrm{CO}_{2}$ dry reforming. Appl. Catal. B, 2015, 164: 100-112.

[73] OEMAR U, HIDAJAT K, KAWI S. Role of catalyst support over PdO-NiO catalysts on catalyst activity and stability for oxy- $\mathrm{CO}_{2}$ reforming of methane. Appl. Catal. A, 2011, 402(1/2): 176-187.

[74] NI J, CHEN L, LIN J, et al. Carbon deposition on borated alumina supported nano-sized $\mathrm{Ni}$ catalysts for dry reforming of $\mathrm{CH}_{4}$. Nano Energy, 2012, 1(5): 674-686.

[75] HAN J, ZHAN Y, STREET J, et al. Natural gas reforming of carbon dioxide for syngas over Ni-Ce-Al catalysts. Int. J. Hydrogen Energy, 2017, 42(29): 18364-18374.

[76] CHEN Q, ZHANG J, PAN B, et al. Temperature-dependent anti-coking behaviors of highly stable $\mathrm{Ni}-\mathrm{CaO}-\mathrm{ZrO}_{2}$ nanocomposite catalysts for $\mathrm{CO}_{2}$ reforming of methane. Chem. Eng. J., 2017, 320: $63-73$.

[77] THEOFANIDIS S A, GALVITA V V, SABBE M, et al. Controlling the stability of a Fe-Ni reforming catalyst: structural organization of the active components. Appl. Catal. B, 2017, 209: 405-416. 